\title{
The Frame Problem and the Semantics of Classical Proofs
}

\author{
G. Graham White \\ Electronic Engineering and Computer Science \\ Queen Mary, University of London \\ London E9 7DB \\ http://eecs.qmul.ac.uk/ graham \\ graham@eecs.qmul.ac.uk
}

\begin{abstract}
We outline the logic of current approaches to the so-called "frame problem" (that is, the problem of predicting change in the physical world by using logical inference), and we show that these approaches are not completely extensional since none of them is closed under uniform substitution. The underlying difficulty is something known, in the philosophical community, as Goodman's "new riddle of induction" or the "Grue paradox". Although it seems, from the philosophical discussion, that this paradox cannot be solved in purely $a$ priori terms and that a solution will require some form of real-world data, it nevertheless remains obscure both what the logical form of this real-world data might be, and also how such data actually interacts with logical deduction. We show, using work of McCain and Turner, that this data can be captured using the semantics of classical proofs developed by Bellin, Hyland and Robinson, and, consequently, that the appropriate arena for solutions of the frame problem lies in proof theory. We also give a very explicit model for the categorical semantics of classical proof theory using techniques derived from work on the frame problem.
\end{abstract}

\section{Background}

\subsection{Circumscription}

Historically one of the first approaches to the frame problem uses a procedure, developed originally by McCarthy [13, called circumscription. We describe it using the outline in Lifschitz 10. As is common in the literature, Lifschitz uses the notion of a fluent, namely a term whose truth value can change over time. ${ }^{1}$ So, the procedure is as follows

1. Select appropriate fluents for the problem: for example, if we are talking about objects moving around, then we might have fluents like at $\left(b_{1}, l_{2}, t\right)$, which says that block $b_{1}$ is at location $l_{2}$ at time $t$.

2. Secondly, "we need to describe first when a combination of values of the frame fluents is 'consistent', that is, [could possibly be] attained in some situation".

3. Next we introduce actions and their postconditions: the postcondition of an action is the set of fluents which become true because it has been executed

\footnotetext{
${ }^{1}$ Many authors in fact use fluents whose value depends on situations, where situations are nodes in the tree of all possible action sequences: we will use the temporally indexed version, because it shows all of the phenomena that we are interested in, it is much easier to align with philosophical work on the grue paradox, and it is notationally much simpler: we have a great deal of notation already, and introducing a tree of situations in addition would be needlessly complicated.
} 
4. There is also the general machinery of the situation calculus in particular the generic law of inertia

$$
\neg \text { noninertial }(f, t) \quad \rightarrow \quad[f(t+1) \leftrightarrow f(t+1)]
$$

(this is a somewhat simplified version of [10, 5.14, p. 333]; here noninertial is a predicate which says that a fluent has changed its truth value at a particular time).

5. Finally we compute the resulting solution by circumscription: we circumscribe - that is, minimise - the extent of the noninertial predicate while holding fixed the postconditions of the actions and obeying the constraints.

\subsubsection{What are Fluents?}

First a question: what are fluents? On the one hand, they look very propositional - they are such things as at $\left(b_{1}, l_{2}, t\right)$ - and, given a fluent $f(\ldots, t)$ and a time $t$, we can obtain a truth-value. So they might, conceivably, be families of propositions parametrised by times (and, indeed, they are called "propositional fluents": [10, p. 328]).

However, the propositional appearance is deceptive: they are, as Lifschitz remarks, "terms and not formulae" [10, p. 328]. We cannot, for example, apply truth-functional connectives to them: in particular, closing them under disjunctions is a good way to make the circumscription mechanism break down. ${ }^{2}$

So fluents have two sides: extensional and intensional. Extensionally, they can be regarded as assignments of truth values to situations, and, regarded in this way, there would be no reason why we should not form arbitrary truth-functional combinations of fluents. Intensionally, however, fluents are terms: we cannot necessarily form arbitrary truth-functional combinations of them.

\subsubsection{The Failure of Uniform Substitution}

There is another way to look at this intensional side. Fluents are typically taken to be literals, and the choice of literals then comes down to the choice of suitable primitives for our language. However, if we do this as a matter of policy, then we cannot choose different primitives: we can have different, but logically equivalent, languages, which yield, on application of the circumscription procedure, different results, precisely because the fluents are different in each case. Thus, a logic formulated using circumscription is not closed under uniform substitution. This is something which has been known for some time, and not usually regarded as a significant problem - see [11]: it is, however, a cause for concern, since it makes our deductions about the world dependent on the primitives that we use to express them in.

And it turns out that the choice of primitives has a considerable influence on the outcome of circumscription: we can show that, by choosing the primitives appropriately, we can (in the propositional case at least) make the outcome of circumscription be anything we want consistent with the axioms and the action postconditions [22. Clearly a great deal is being smuggled in under the choice of primitives, and it would be good to have some more mathematical insight into what the choice amounted to.

\footnotetext{
${ }^{2}$ We may, of course, define what Shanahan calls "compound fluents" [16 pp. 115f.], and we can define recursive conditions for $f(\ldots, s)$ to be true when $f$ is such a fluent. However, these fluents play no role in the minimisation procedure; and it is this role in the minimisation procedure that we are concerned with.
} 


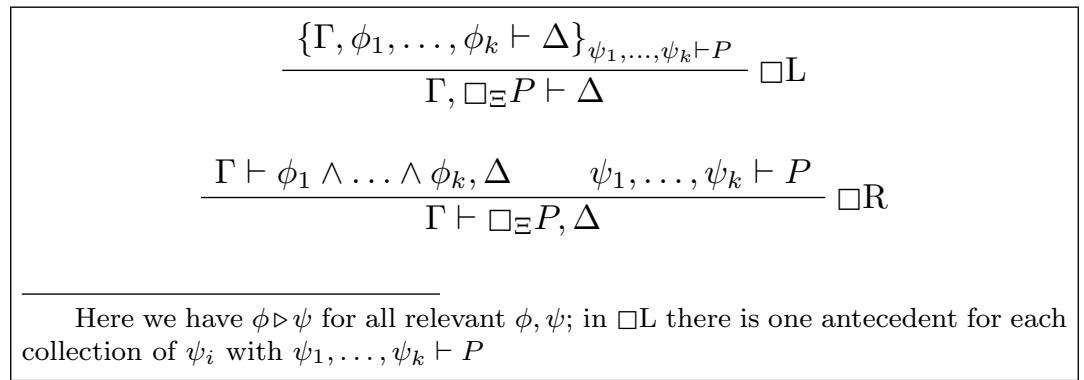

Table 1: Proof Rules for the McCain-Turner Modals

\subsection{McCain and Turner}

McCain and Turner [12, 21 found out how to reformulate the circumscription procedure in a mathematically more perspicuous way. We give ourselves a language $\mathcal{L}$, together with a set $\Xi$ of clauses, which I shall write in the form

$$
\phi \triangleright \psi
$$

Here $\phi$ and $\psi$ are propositions, time-varying in the case of the frame problem. McCain and Turner have a model-theoretic definition of inference with these clauses: it is equivalent to the following.

First we define a modal operator $\square_{\Xi}$, depending on $\Xi$ : it will be the strongest $\mathbf{K}$ modal operator on $\mathcal{L}$ for which all of the entailments

$$
\phi \vdash \square \psi \quad \text { for all } \phi \triangleright \psi
$$

are theorems. We have given a proof theory for this operator which satisfies cut elimination [23, 24]; the left and right rules for the modal operators are Table 1. We then add to our theory the set of axioms $p \leftrightarrow \square p$, for all propositions $p$.

Remark 1. Notice that cut elimination is important here: prima facie the left rule has an infinite number of antecedents, but (if the clauses are well enough behaved) an argument analogous to the subformula property shows that a finite number of them suffice.

Remark 2. Although the original application to the frame problem only requires clauses with non-modal members, the proof of cut elimination applies to the case where we define wellfounded sequences of modalities by means of clauses involving modalities earlier in the sequence. This will be important for our results on the semantics of classical proofs.

We can regard this logic as describing a certain sort of explanation: the clauses in $\Xi$ can be regarded as basic explanations, and an entailment

$$
p \vdash \square q
$$

can be read as " $p$ explains $q$ ". And, when we add the axioms $p \leftrightarrow \square p$, we are endeavouring to construct an explanatorily closed theory: that is, we have a language which contains not just propositions, but explanations of those propositions, and we want a description of the world in which every fact has an explanation and in which every explanation guarantees the truth of the explained fact. We will, throughout this paper, use a terminology of explanation in describing the McCain-Turner theory, rather than, as they do, using a terminology of causality: in particular, sets of clauses will be called explanatory, rather than causal, theories. 
There will be two types of propositions here, fluents and actions: We write fluents and actions with a temporal index, but this is not part of the formal syntax(we cannot quantify over the indices, for example).

First, we give initial conditions at $t=0$ : that is, for fluents or negations of fluents $p_{0}$ at time 0 , we have

$$
\triangleright p_{0}
$$

next, we say that, if an action occurs at $t$, it explains its postconditions at $t+1$ : so, if $p$ is the postcondition of $\alpha$, we have

$$
\alpha_{t} \triangleright p_{t+1}
$$

for all $t$. Finally, we give the frame axiom: for all fluents $\phi$, we have

$$
\phi_{t} \wedge \phi_{t+1} \triangleright \phi_{t+1}
$$

or, in other words, if a fluent is true at $t$, and its truth value is unaltered between $t$ and $t+1$, that is sufficient explanation for its truth at $t+1$ (in other words, for fluents persistence is self-explanatory).

Table 2: McCain and Turner's "Causal" Rules

So much for the general theory. We apply it to the frame problem by considering sets of clauses like those in Table 2 (see [12]).

This is something of an improvement over circumscription, because the action takes place in a monotonic modal theory: the only part of the machinery which is nonmonotonic turns out to be that, if you add more clauses to $\Xi$, you can end up with fewer valid inferences than you started with (because of the left rule for $\square \Xi$, or the right rule for $\mho_{\Xi}$ ). And generally the logic is better behaved: we can prove cut elimination, and cut elimination leads to the possibility of proof search.

However, there is still one part of this construction which is not obviously invariant under uniform substitution: that is, the choice of fluents. Arguably we have still made progress, because the fluents figure in the clauses defining the basic explanations, and we use the basic explanations as a presentation of the modal operator, which defines our notion of (non-basic) explanation. And, in general, there will be different choices of fluents which give rise to the same modal operator, and so the McCain-Turner formulation is not as brutely nonextensional as the standard formulation of circumscription. However, as the examples in [22] show, there are choices of fluents which do change the outcome of circumscription, and so, if we reformulate this circumscription using McCain-Turner's formalism, we get choices of clauses which do change the modal operator (since the modal operator determines the predictions in the McCain-Turner formalism). So, clearly, there is something here still to be investigated.

\subsection{The Grue Paradox}

We have, then, reduced the frame problem to a single question: that of finding fluents, i.e. timedependent primitives for which persistence is explanatory. Now if we transpose this problem 
into the terms of the problem of prediction, we get the following question: for which temporally parametrised propositions can observation license prediction? In this formulation, the problem has been known in the philosophical community, and is officially referred to as Nelson Goodman's "New Riddle of Induction", and more colloquially as the "grue paradox". 19, 18, 8, Ch. III] 14

Abstractly, the problem can be described as follows. Suppose that we have a language with two temporally indexed propositions, $\phi$ and $\psi$, and that $\phi$ and $\psi$ are contradictory (i.e. that, for all $\left.t, \phi_{t} \wedge \psi_{t} \vdash \perp\right)$. Supoose also that we can successfuly predict $\phi$ and $\psi$ by observation: that is, that the observation of $\phi_{t}$ warrants the prediction of $\phi_{t+1}$, and similarly for $\psi$. Then, for some $t_{0}$, define ${ }^{3}$

$$
\begin{aligned}
& \tilde{\phi}= \begin{cases}\phi & \left(t<t_{0}\right) \\
\psi & \left(t \geq t_{0}\right)\end{cases} \\
& \tilde{\psi}= \begin{cases}\psi & \left(t<t_{0}\right) \\
\phi & \left(t \geq t_{0}\right)\end{cases}
\end{aligned}
$$

We note the following:

1. If $\phi$ and $\psi$ warrant prediction, then $\tilde{\phi}$ and $\tilde{\psi}$ cannot, because the two pairs make contradictory predictions

2. The relation between the $\langle\phi, \psi\rangle$ pair and the $\langle\tilde{\phi}, \tilde{\psi}\rangle$ pair is entirely symmetric: the former can be defined from the latter by means of definitions with entirely the same form as the definitions above

3. We can, then, regard the language in which these definitions are formulated in two ways: one in which $\langle\phi, \psi\rangle$ are primitive and in which $\langle\tilde{\phi}, \tilde{\psi}\rangle$ are defined, and vice versa. These two formulations are indistinguishable from the point of view of logical form

4. Attempts to say that $\langle\tilde{\phi}, \tilde{\psi}\rangle$ are somehow "artificial" because their definitions involve an arbitrary time are question-begging: their definitions only involve an arbitrary time in a language in which the rival concepts are primitive

5. Similarly, attempts to say that our mental concepts, or the recognitional capacities exercised by our senses, legitimate $\langle\phi, \psi\rangle$ rather that $\langle\tilde{\phi}, \tilde{\psi}\rangle$ are likewise question-begging: how do we know that the concept we now deploy is $\phi$ or $\tilde{\phi}$ ?. For this reasons, attempts such as this are liable to be received with scorn by anyone familiar with the literature on Wittgenstein's Private Language Argument

The grue paradox, then, is non-trivial. The more or less received philosophical position is that, in order to predict change, one needs to have a set of primitives which, in the absence of causes, will persist unchanged: such primitives are called projectibles. Sets of projectibles cannot be chosen on purely logical grounds, but must, in some way, reflect the causal structure, or ontology, of the world; that is what the grue paradox shows.

\subsection{McCain-Turner and the Grue Paradox}

We should note that the grue paradox, traditionally stated, is about the licensing of inference by projectibles, whereas, as we have argued, the McCain-Turner formalism is about the licensing

\footnotetext{
${ }^{3}$ Traditionally, $\tilde{\phi}$ is known as grue, and $\tilde{\psi}$ as bleen.
} 
of explanation by projectibles. The basic pattern is that, if $P$ has the same truth value at 0 and 1 , and $P$ is true at 0 , then we have an explanation of $P$ being true at 1 . Explanation seems in any case to be more delicate: for example, logically valid inference seems to be closed under disjunction, whereas explanatory projectibles are clearly not.

We have, so far, a language consisting of temporally indexed propositions, of which there are two sorts, actions and fluents. We can define new primitives in a three-sorted language: there will be times $\left(t_{0}, t_{1}, \ldots\right)$ and trajectories, which will intuitively speaking be $n$-tuples of fluent literals $\lambda=\left(f_{0}, f_{1}, \ldots\right)$ (supposed finite for simplicity). We can recover a temporally indexed proposition from a trajectory and a time: $\lambda \wedge t_{i}$ will be some temporally indexed proposition $P_{i}$. Similarly, we can define times: $t_{0}$ will be $f_{0} \vee f_{0}^{\prime} \vee f_{0}^{\prime \prime} \ldots$, where we make a disjunction of all of the fluent literals indexed with 0 . And we can make a trajectory from a series of fluent literals: the trajectory will be of the form $\left(t_{0} \rightarrow f_{0}\right) \wedge\left(t_{1} \rightarrow f_{1}\right) \wedge \ldots$ So the language of trajectories plus times is equivalent to the simple propositional language that we started with. Using this equivalence, we can define operations on trajectories, using merely the logical operations on propositions: we can modify a single temporal value of a trajectory, or, given two trajectories and a time, we can graft the two trajectories together: $\lambda_{1}$ before $t, \lambda_{2}$ at and after $t$. We can, thus, start with a set of primitive trajectories and construct the others from them: in this way, the two alternative futures for the frame problem correspond to different choices of primitive trajectories.

We can now rewrite the McCain-Turner set of rules in terms of trajectories. The initial conditions are no trouble: they are all of the form $\triangleright\left(t_{0} \rightarrow \lambda\right)$, for suitable $\lambda$. Similarly, the action postconditions will all be of the form $(t \rightarrow a) \triangleright(t+1 \rightarrow p)$, for suitable $t, a$ and $p$. The persistence axioms, however, will all be of the form

$$
(t \rightarrow \lambda) \wedge(t+1 \rightarrow \lambda) \triangleright(t+1 \rightarrow \lambda)
$$

and, simply by varying the primitive trajectories, we get one outcome or the other for the grue paradox. So we have shown:

$$
\begin{aligned}
& \text { The McCain-Turner system reduces the frame problem to the grue para- } \\
& \text { dox. }
\end{aligned}
$$

In this context, the McCain-Turner system seems to be rather important: it has swept away a large amount of what appear to be ad hoc constructions and focussed on a philosophically significant issue. We shall now concentrate on that issue.

\section{The Semantics of Proofs}

In this section, we will show that the McCain-Turner calculus can be applied to the semantics of classical proofs: this will, in turn, give us a proof-theoretic approach to the frame problem.

\subsection{Background}

Bellin et al. 22 give a proof theory for the classical propositional sequent calculus. We start with a rather formal issue: there are basically two approaches to a categorical formulation of a sequent calculus. One is to consider a sequent $\Gamma \vdash \Delta$ as a proof of $\bigvee \Gamma \vdash \wedge \Delta$, to regard $\bigvee \Gamma$ and $\Lambda \Delta$ as objects in a category, and then to investigate the structure of that category. The other approach is to formalise more directly the notion of a morphism from one collection of objects to another collection: this yields what is called a polycategory [20]. Bellin et al. [2] show 
that the two approaches (let us call them the categorical and the polycategorical approaches) are systematically related: given a morphism $f: X \rightarrow Y$ from the categorical formulation, we can recover contexts (i.e. the sets of formulae $\Gamma$ and $\Delta$ ) from it by defining suitable idempotent endomorphisms of $X$ and $Y$, splitting them, and then showing that their domains have enough structure to yield a polycategory.

This is a somewhat abstract formulation, but it shows the importance of idempotent proofs. Now we can easily get idempotent proofs out of the McCain-Turner formalism: a simple example is "obvious" proof of

$$
\square \Xi X \vdash \square \Xi X
$$

(that is, the one which first applies $\square \mathrm{L}$ and then, on each premise, the corresponding instance of $\square \mathrm{R}$ ). Cut elimination on the composition of (5) with itself yields (5); so, the explicit construction of idempotents is extremely easy in the McCain-Turner formalism, and we will consequently, find that it has something to say about the categorical semantics of classical proofs (although, as it happens, the idempotents that we will use will not be these ones).

Furthermore, we have an equivalence

$$
\begin{gathered}
\square=\cong \quad \bigvee_{\substack{\phi_{1}, \ldots, \phi_{k} \vdash X \\
\phi_{i} \triangleright \psi_{i}}} \phi_{1} \wedge \ldots \wedge \phi_{k} \\
\\
\end{gathered}
$$

so that $\square \Xi X$ is a disjunction (and, given $\Xi$, canonically so, with the disjuncts indexed by conjunctions of members of $\Xi$ ). Given our reading of the McCain-Turner theory in terms of explanation, we can also regard the right hand side of (6) as a paraphrase of $X$ in a distinguished explanatory vocabulary, namely the vocabulary given by the primitives $\phi_{i}$.

Remark 3. The standard approach to logic is to consider propositions as built up from so-called "primitives" using connectives. Usually, however, we undo the dependence on a particular set of primitives by insisting on invariance under uniform substitution. The above approach does not do this: $X$ and $Y$ are given, but they do not need to be given together with any syntactic structure. Syntactic structure is imposed on them by means of the modal operators, which can be regarded as analytical instruments: but there are many different sets of clauses which will give the same modal operators, and each of these choices will give a different concrete syntax. However, if the modal operators are the same, then the semantics (and, in some sense, the abstract syntax) will be the same.

This is somewhat the same as Girard's approach in [7, ch. 13] and [6], where he considers logical systems where the process of rule application (and, correspondingly, the process of syntactic decomposition) goes on for ever: one never reaches a foundational level of syntactic primitives (see also Schleiermacher [15, who develops logical techniques for reconciling disagreeing parties in a dispute, a situation where it would be overly optimistic to hope for a priori agreement on vocabulary).

\subsection{Kleisli Categories}

We can define a sequent calculus on the basis of these operators by means of a Kleisli construction. First, some terminology. As well as the $\square$ operator which we have just defined, we will also define a $\diamond$ operator by a dual definition:

Definition 1. Given a set of explanatory clauses $\Xi$, we define an operator $\diamond_{\Xi}$ by the following 
left and right rules:

$$
\begin{gathered}
\frac{\Gamma, \psi_{1} \wedge \ldots \wedge \psi_{k} \vdash \Delta \quad P \vdash \phi_{1}, \ldots, \phi_{k}}{\Gamma, \diamond_{\Xi} P \vdash \Delta} \diamond \mathrm{L} \\
\frac{\left\{\Gamma \vdash \psi_{1}, \ldots, \psi_{k}, \Delta\right\}_{P \vdash \phi_{1}, \ldots, \phi_{k}}}{\Gamma \vdash \diamond_{\Xi} P, \Delta} \diamond \mathrm{R}
\end{gathered}
$$

We should note that this modality is not $\neg \square \neg$, but is, rather, the adjoint of $\square$ :

Lemma 1. If $\Xi$ is any set of rules, then $\square \Xi$ and $\nabla_{X i}$ are adjoint: i.e. we have

$$
X \vdash \square_{\Xi} Y \quad \text { iff } \quad \diamond_{\Xi} X \vdash Y
$$

Now consider a sequent like

$$
\diamond_{\Xi} X \vdash \square_{\Pi} Y
$$

it is an entailment between a pair of propositions, and can be regarded as a morphism in the categorical formulation. The modal operators, however, define idempotent proofs, and, when we split these idempotents, we get conjunctions and disjunctions which give a sequent version of (7) (and it is easy to show that the disjuncts are the same as the disjuncts in (6), and dually for the conjuncts on the left). So we can use these modal operators to exhibit the polycategorical structure of sequent calculus proofs.

The sequent above looks like a morphism in the Kleisli category of $\square$. However, the Kleisli construction requires monads (or, dually, comonads) - in the present context, it requires S4 modalities - but the normal McCain-Turner modalities are $\mathbf{K}$ rather than $\mathbf{S} 4$. However, rule sets with certain properties will yield $\mathbf{S} \mathbf{4}$ modalities.

Trivially, if all the rules in a set are of the form $\phi \triangleright \phi$, then we get a $\mathbf{S 4}$ modality. Call such rules self-explanatory; self-explanatory rules are, in fact, enough to give us a proof theory for classical propositional calculus, but they will not be enough for applications to the frame problem.

There are two components that we need to add: one is the rule $\mathbf{T}$ (i.e. $\square P \vdash P$ ), and to get this we need rules $\phi \triangleright \psi$ such that $\phi \vdash \psi$. Call such rules reflexive. Secondly, we need the rule 4, that is, $\square P \vdash \square \square P$, and for this we need a transitivity condition on the clauses.

First some notation. Suppose we have a tuple of clauses $\phi_{1} \triangleright \psi_{1}, \ldots, \phi_{k} \triangleright \psi_{k}$; we can write such a tuple as $\vec{\phi} \triangleright \vec{\psi} . \wedge \vec{\phi}$ will stand for $\phi_{1} \wedge \ldots \phi_{k}$, and similarly for $\wedge \vec{\psi}, \vee \vec{\phi}$, and so on; similarly, a sequent $\vec{\psi} \vdash \Delta$ will mean the sequent $\psi_{1}, \ldots, \psi_{k} \vdash \Delta$, and similarly for the obvious variants. $\vec{\phi} \triangleright \vec{\psi} \in \Xi$ means that each $\phi_{i} \triangleright \psi_{i} \in \Xi$. If we have a tuple of tuples, then $\vec{\phi}_{i}$ will stand for the relevant $i$ th tuple.

Definition 2. A set $\Xi$ of rules is transitive if, whenever we have $\vec{\phi} \triangleright \vec{\psi} \in \Xi$, and if we have $\vec{\psi} \vdash \square \Xi Y$ for some $Y$, then we have $\phi \vdash \square \Xi Y$.

This can be reformulated in terms of the $\diamond$ modality:

Lemma 2. A set of rules is transitive if, equivalently, whenever $\vec{\phi} \triangleright \vec{\psi}$, and $\diamond X \vdash \vec{\phi}$, then $\diamond X \vdash \vec{\psi}$

And so we have 
Proposition 1. $\square \Xi$ is an $\boldsymbol{S}_{4}$ modality iff $\Xi$ is a reflexive transitive set of rules iff $\rangle_{\Xi}$ is an $\boldsymbol{S}_{4}$ modality.

Example 1. If we split $P$ using the explanatory theory with the single explanatory law $P \triangleright P$, we get the context with a single proposition $P$ : if we split $P \vee Q$ using the theory with explanatory laws $P \triangleright P$ and $Q \triangleright Q$, we get the right context $P, Q$. Both of these rule sets are self-explanatory, and so reflexive and transitive.

We will also require another condition on rule sets in order to keep our proofs finite. As we have said, we can still prove cut elimination if we allow clauses to contain modalised subformulae, provided that there are no circular dependencies between modalities. In addition, we want a proof involving $\square$ on the right, for example, not to involve the infinitary left rules for subordinate $\square$ modalities. The following condition guarantees this:

Definition 3. A clause $\phi \vdash \psi$ is well-balanced if $\psi$ is either non-modal or it is some $\square_{\Pi} Y$, and dually for $\phi$. A modality $\square_{\Pi}$ or $\nabla_{\Xi}$ is well balanced if all the clauses in its rule set are; and a modality is hereditarily well balanced if it is well balanced and all the modalities occurring in its clauses are hereditarily well balanced.

\subsection{A Classical Category}

Consider, then, entailments

$$
\diamond_{\Xi} X \quad \vdash \quad \square_{\Pi} Y
$$

where $\Xi, \Pi$ are self-explanatory sets of rules. We will, in this section and the following, work exclusively with such rules. We would like to regarded such an entailment as a morphism in a suitable Kleisli category (this is naturally equivalent to the formalism with $X \vdash \square_{\Pi^{\prime}}$, for suitable $\Pi^{\prime}$ ). We will, then, construct a category out of entailments such as (8), together with the usual apparatus of Bellin et al [2]: we will give a guarded functor from the guarded category of classical proofs to our category. The linear idempotents will be proofs of $\mho_{\Xi} P \vdash \square \Xi P$.

So, the definition ought to go something like this:

Definition 4. We define a category, the McCain-Turner category, as follows:

Objects are non-modal formulae $X, Y \ldots$;

Morphisms are cut-free proofs of $\mho_{\Xi} X \vdash \square_{\Pi} Y$, for any $\Xi, \Pi$.

Composition This comes from cut elimination of the chain of proofs

$$
\diamond_{\Xi} X \vdash \square_{\Pi} Y \vdash Y \vdash \diamond_{\Xi^{\prime}} Y \vdash \square_{\Pi^{\prime}} Z
$$

There are two problems with this: one is that we have not defined identity of morphisms, and the other, related problem is that we have not addressed the possible nondeterminacy of cut elimination.

Consider these modalities $\mho_{\Xi}, \square_{\Pi}$, and so on. We are interested not so much in the modalities themselves - although they are, basically the same as the boxes that occur in modern proof theory - but we are, rather, interested in their presentation by means of the sets of clauses $\Xi$, $\Pi$ (these are presentations somewhat in the style of Lack [9]). It is these sets of clauses which carry the interesting structure of the classical category: and what they do is to present the $\mathbf{S 5}$ modalities (i.e. monads and comonads) from which we make the model of classical propositional logic.

Here are some phenomena which may show that this problem of the identity of morphisms is not trivial. 
Kleisli Equivalences It is trivial that, if we have an adjoint monad and comonad, then the category with morphisms $\diamond A \rightarrow \square B$ is (by adjunction) equivalent to the category with morphisms $\diamond A \rightarrow \square \square B$, which is (by the monad laws) naturally equivalent to the coKleisli category of $\square$. This holds for a single monad: however, if (as we will have) we have many adjoint monad-comonad pairs, and we try to prove a similar result, we find a category with morphisms $A \rightarrow \square^{\prime} \square B$, but $\square^{\prime} \square$ is not a comonad (though in our case it can be completed to one). Furthermore, the relation between presentations of the $\diamond^{\prime}, \square$ pair and presentations of the monad generated by the completion of $\square^{\prime} \square$ is a little opaque.

Composition of Morphisms We get a range of similar problems here, which are presumably related to the nondeterminacy of cut elimination.

Global Questions Even if we can solve these problems morphism by morphism, can we do so globally (either for all elements of a homset, or for an entire classical category)? Are their naturally occurring structures which look, locally, just like classical categories, but which do not have any global presentation by proofs in a single language? ${ }^{4}$

Now ad hoc solutions can be given for some cases of these problems of identity and naturality: however, it is not clear that these solutions are very principled. A principled solution may well need a 2-categorical justification: to ignore this may well be to ignore, also, the sort of delicate issues which make the semantics of classical proofs so difficult.

With these reservations in mind, here is a definition which appears to work for representing classical categories: it is somewhat crude, and it is not at all clear that it is the only (or the best) definition possible.

First a lemma on the form of proofs of the sequents in question.

Lemma 3. A cut-free proof of

$$
\diamond_{\Xi} X \vdash \square_{\Pi} Y
$$

where the modalities are hereditarily well balanced, can, after suitable rule permutation, be reduced to a proof of

$$
\vee \vec{\psi}_{1}, \ldots, \vee \vec{\psi}_{k} \vdash \wedge \vec{\phi}_{1}^{\prime}, \ldots, \wedge{\overrightarrow{\phi^{\prime}}}_{l}
$$

followed by applications of $\diamond_{\Xi} L, \square_{\Pi} R$, and contractions.

Proof. Because the modalities are hereditarily well balanced, we know that the proof is finitely branching and hence finite. Rewrite the proof from above as follows: ignore all modal rules (which must be either $\square \mathrm{R}$ or $\diamond \mathrm{L}$ ) for the topmost modalities and all contractions on modal formulae. We are left with a sequent of the required form, which proves the conclusion by the application of the delayed modal rules followed by contractions.

Definition 5. Consider the collection of cut free proofs of

$$
\diamond_{\Xi} X \vdash \square_{\Pi} Y
$$

and suppose that the modalities in question are hereditarily well balanced.

By Lemma 3, such a cut free proof can be transformed into a proof of

$$
\vee \vec{\psi}_{1}, \ldots, \vee \vec{\psi}_{k} \vdash \wedge \vec{\phi}_{1}^{\prime}, \ldots, \wedge \vec{\phi}_{l}^{\prime}
$$

\footnotetext{
${ }^{4}$ Bear in mind Schleiermacher and his project of explaining how people can come to agreement even if they do not initially share a vocabulary 15
} 
followed by applications of the left and right rules for $\nabla_{\Xi}$ and $\square_{\Pi}$, respectively. If we then cut (11) with the obvious proofs of $\psi_{i j} \vdash \vee \vec{\psi}_{i}$ and of $\wedge{\overrightarrow{\phi^{\prime}}}_{i^{\prime}} \vdash \phi_{i^{\prime} j^{\prime}}^{\prime}$, we get a matrix of sets of proofs of the matrix of entailments $\left(\psi_{i, j} \vdash \phi_{i^{\prime} j^{\prime}}^{\prime}\right)$ (sets of proofs because cut elimination is nondeterministic). If the matrix of entailments is entirely nonmodal, then we stop there; if not, then some of the $\psi$ or of the $\phi^{\prime}$ are either $\diamond_{\tilde{\Xi}} \tilde{X}$ or $\square_{\tilde{\Pi}} \tilde{Y}$, respectively. In this case we keep on, recursively: we obtain a matrix (possibly with some nested substructure), indexed by occurrences of antecedents and succedents of explanatory clauses, of proofs of nonmodal entailments. And we say that any two proofs of entailments with the same indices are the same (so that we get complexly indexed proofs whose entries are booleans), and we say that two proofs of (11) are the same if they have the same matrix of booleans.

The similarity of this definition to constructions in the geometry of interaction, and its dependence on those constructions, ought to be clear.

Theorem 1. The McCain-Turner category is a static model for classical propositional logic as defined in [2, Definition 3.23].

Proof. The structure is as follows. Note that the data are functors and natural transformations: we will give generic morphisms, and guarded functoriality and guarded naturality are straightforward, but long-winded, to verify. Since our clauses here will all be self-explanatory, we shall, in this section, write $\phi$ instead of $\phi \triangleright \phi$.

Duality Our data are all clearly self-dual

Connectives $T$ corresponds to the proposition $T$ together with the proof

$$
\frac{\overline{\nabla_{\emptyset} \top \vdash \top}}{\nabla_{\emptyset} \top \square_{\emptyset} \top} \square \mathrm{R}
$$

where $\square \mathrm{R}$ uses the empty sent of fluents; this proof is the guard, and is, rather degenerately, idempotent under the defined composition.

$\wedge$ of two proofs

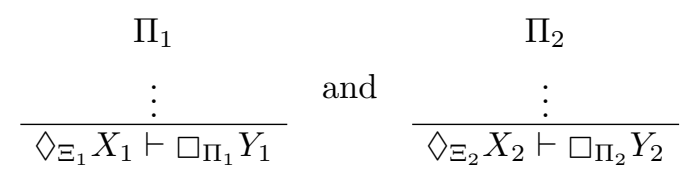

is

$$
\begin{aligned}
& \Pi_{1} \quad \Pi_{2} \\
& \frac{\frac{\vdots}{\oslash_{\Xi_{1}} X_{1} \vdash \square_{\Pi_{1}} Y_{1}}-\frac{\vdots}{\diamond_{\Xi_{1}, \Xi_{2}}\left(X_{1} \wedge X_{2}\right) \vdash \square_{\Pi_{1}} Y_{1} \wedge \square_{\Pi_{2}} Y_{2}}}{\diamond_{\Xi_{2} Y_{2}}}
\end{aligned}
$$

We here impose the condition that the sets $\Xi_{1}, \Pi_{1}$ are disjoint from each other: we can do this by suitable renaming of the propositions in them. 
Linear Idempotents We start with the morphisms

$$
\frac{\overline{P \vdash P}}{\diamond_{P} P \vdash \square_{P} P} \diamond \mathrm{L}, \square \mathrm{R}
$$

and close under $\wedge$ and $\vee$.

Unit Law and Associativity of $\wedge$ Routine calculation: we need a guarded natural transformation, and we can verify that the squares commute in the guarded sense.

Symmetry Clear: our data is invariant under symmetry.

Linear Distributivity The proof is

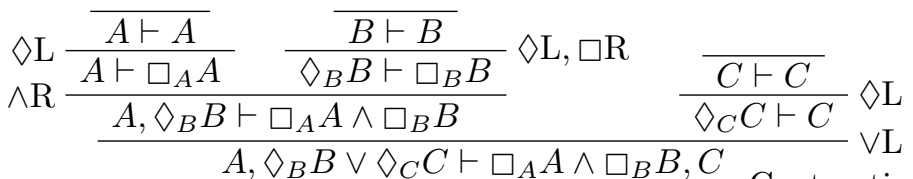

$$
\begin{aligned}
& \frac{A, \diamond_{B} B \vee \diamond_{C} C \vdash \square_{A} A \wedge \square_{B} B, C}{\diamond_{A, \diamond_{B} B \vee \diamond_{C} C} X \vdash \square_{\square_{A} A \wedge \square_{B} B, C} Y} \text { Contraction, } \diamond \mathrm{L}, \square \mathrm{R}
\end{aligned}
$$

(here $X$ is $A \wedge(B \vee C)$ and $Y$ is $(A \wedge B) \vee C)$.

$\eta$ Similar to linear distributivity

Monad, Comonad The comonad structure for $\wedge$ is given by proofs

$$
\frac{\frac{\overline{A \vdash A}}{\diamond_{A} A \vdash \square_{A} A} \diamond \mathrm{L}, \square \mathrm{R} \quad \frac{\overline{A \vdash A}}{\diamond_{A} A \vdash \square_{A} A}}{\diamond_{A} A \vdash \square_{A} A \wedge \square_{A} A}{ }^{\diamond_{A} A \vdash \square_{\square_{A}} A \wedge \square_{A} A} \square \mathrm{R}, \square \mathrm{R}
$$

and

$$
\frac{\overline{\diamond_{A} A \vdash \top}}{\diamond_{A} A \vdash \square_{\emptyset} \top} \diamond \mathrm{R}
$$

We can, on the basis of this definition, construct a functor from the classical category of proofs to our classical category; the definitions are mostly straightforward, and have been omitted for lack of space.

\section{The Frame Problem}

We can now tackle the frame problem: we will revert to working with general rules, but we will require reflexive and transitive rule sets. The clauses will not have any nested modal structure, so defining identity of morphisms will be fairly simple.

Consider the McCain-Turner set of clauses given in Table 2. This is almost reflexive: the only non-reflexive part of it are the clauses $\triangleright P$ for propositions stipulated to be true at the starting state. We can simply neglect these: they seem to be an attempt to put boundary 
conditions into the laws of the theory. The remainder is reflexive, and we can make it transitive by closing under application of the transitivity conditions.

This is what we get. Define a partial trajectory as follows (see the definition of a trajectory on p. 420. We assume discrete time.

Definition 6. A partial trajectory is a conjunction of fluents at times within a given temporal interval, $\left[t_{A}, t_{B}\right]$, such that

1. At each time $t$ in this interval, there is a conjunct of the form $t \rightarrow f_{t}$, for some fluent literal $f_{t}$

2. There may also be conjuncts of the form $t \rightarrow a_{t}$, for action literals $a$

3. if $t$ is in the interval and before the endpoint, then

either there is no action literal at $t$, and we have conjuncts $\left(t \rightarrow f_{t}\right) \wedge\left(t+1 \rightarrow f_{t+1}\right)$

or there is an action literal $a$ at $t$, and we have conjuncts $\left(t \rightarrow f_{t} \wedge a_{t}\right) \wedge\left(t+1 \rightarrow g_{t+1}\right)$ where $g$ is the postcondition of $a$

A trajectory $\tau$ is the truncation of a trajectory $\tau^{\prime}$ if

1. $\tau$ is defined over $\left[t_{A}, t_{B}\right]$

2. $\tau^{\prime}$ is defined over $\left[t_{A}, t_{B^{\prime}}\right]$, with $t_{B}<t_{B^{\prime}}$, and

3. $\tau^{\prime}$ restricted to $\left[t_{A}, t_{B}\right]$ is $\tau$.

Then we have

Proposition 2. The transitive closure of the McCain-Turner rules is the set of partial trajectories, with $\tau^{\prime} \triangleright \tau$ whenever $\tau$ is a truncation of $\tau^{\prime}$.

Call this set of rules $\mathcal{M}$. Consider now sequents of the form

$$
\diamond_{\mathcal{M}}\left(t_{A} \rightarrow f_{t_{A}}\right) \tau \vdash \square_{\mathcal{M}}\left(t_{B} \rightarrow f_{t_{B}}^{\prime}\right)
$$

where $T$ is the transitive closure of the McCain-Turner rules and where $\tau$ is some trajectory. The antecedent is the conjunction of the set of all partial trajectories which are explained by $\tau$, i.e. the set of all truncations of $\tau$. The consequent is the set of all explanations of $f^{\prime}$ at $t_{B}$, i.e. the set of all trajectories which end with $f^{\prime}$ at $t_{B}$. So, if we do an abductive proof search for proofs of this sequent, we can find the possible causal histories which terminate with $f^{\prime}$ at $t_{B}$.

So there is a sense in which, in this case, the modalities impose a language of partial causal histories on our data: if $X$ and $Y$ represent, for example, states of the world, then the modalities make them communicate by translating them into partial causal histories. This gives a more structured description of the data necessary for a resolution of the grue paradox. These modalities $-\square \Xi$ and its adjoint modality - describe how general propositions are translated into sets of partial causal histories: they specify what could be called an ontology (an ontology in the philosophical sense, not in the sense of the semantic web). This is vastly more meaningful than the "sets of projectibles" which have previously been used as a solution of this paradox: furthermore, we have a criterion for saying when sets of projectibles are equivalent, compatible, and so on (we simply expand them into transitive reflexive sets of clauses, and see whether the resulting modalities are the same or not). 


\section{Loose Ends}

There are a great number of technical points still unresolved. We have shown that a great deal of the proof theory of classical propositional calculus can be represented by explicit calculations with McCane-Turner modalities: this is interesting, but leaves scope for future work. There are obvious connections between this work and the geometry of interaction (one can, for example, write down a traced monoidal structure on the McCain-Turner category, and, from that viewpoint, a comparison with Abramsky [1, and, as should be clear, there are a good number of detailed connections with Girard's fascinating work on the subject.)

There are also possible connections with machine learning. Smale and Zhou [17] have an analysis of machine learning in terms of two Hilbert spaces, one of them being a dense subspace of the other. Points in the "larger" Hilbert space represent states of the world, and are unknown: it may, for example, be a space of functions, and we are trying to find an approximation to it by sampling its output for a finite set of random inputs. The smaller Hilbert space is a space of parametrised functions with which we are trying to approximate the function that we are sampling: it is often called the hypothesis space. What we do is to make a least squares approximation to the tuple of sample values using a member of the hypothesis space: that is, we project from the space of sample tuples to the hypothesis space according to a particular metric.

This has at least a superficial resemblance to our project in this paper (we have an explanatory, descriptive "vocabulary" given by points in the hypothesis space, and we are trying to use this vocabulary with a view to inference about the space that the hypothesis space represents). But there is possibly more to it than that: Girard has encodings in Hilbert and Banach spaces [5, 3, 4] which could represent most of the constructions that we have performed in this paper. And, if anything were to come of this, it might yield some sort of correspondence between machine learning and proof theory.

\section{References}

[1] Samson Abramsky, Esfandiar Haghverdi, and Philip J. Scott. Geometry of interaction and linear combinatory algebras. Mathematical Structures in Computer Science, 12(5):625-665, 2002.

[2] Gianluigi Bellin, Martin Hyland, Edmund Robinson, and Christian Urban. Categorical proof theory of classical propositional calculus. Theoretical Computer Science, 364(2):146 - 165, 2006. Logic, Language, Information and Computation, 11th Workshop on Logic, Language, Information and Computation.

[3] Jean-Yves Girard. Geometry of interaction 2: deadlock-free algorithms. In Per Martin-Löf and Grigori Mints, editors, Conference on Computer Logic, volume 417 of Lecture Notes in Computer Science, pages 76-93. Springer, 1988.

[4] Jean-Yves Girard. Geometry of interaction (abstract). In Bengt Jonsson and Joachim Parrow, editors, CONCUR, volume 836 of Lecture Notes in Computer Science, page 1. Springer, 1994.

[5] Jean-Yves Girard. Coherent banach spaces: a continuous denotational semantics. Electr. Notes Theor. Comput. Sci., 3:81-87, 1996.

[6] Jean-Yves Girard. Locus solum: From the rules of logic to the logic of rules. In Laurent Fribourg, editor, Computer Science Logic, volume 2142 of Lecture Notes in Computer Science, pages 38-38. Springer Berlin / Heidelberg, 2001.

[7] Jean-Yves Girard. Le Point Aveugle: Cours de Logique. Hermann, Paris, 2006.

[8] Nelson Goodman. Fact, Fiction and Forecast. Harvard University Press, fourth edition, 1983. 
[9] Stephen Lack. A 2-categories companion. Available online at http://arxiv.org/abs/math/ $0702535 v 1,2007$.

[10] Vladimir Lifschitz. Circumscription. In Handbook of Logic in Artificial Intelligence and Logic Programming, volume 3, pages 297-352. Clarendon, 1994. Volume Coordinator D. Nute.

[11] David Makinson. Ways of doing logic: What was different about AGM 1985? Journal of Logic and Computation, 13(1):3-13, 2003.

[12] Norman McCain and Hudson Turner. Causal theories of action and change. In Proceedings of AAAI97, pages 460-465, Providence, RI, 1997. AAAI.

[13] John McCarthy. Circumscription - a form of non-monotonic reasoning. Artificial Intelligence, 13:27-39, 1980.

[14] Hilary Putnam. Foreward. In Fact, Fiction and Forecast 8], pages vii-xvi.

[15] Friedrich Daniel Ernst Schleiermacher. Dialektik. Wissenschaftliche Buchgesllschaft, Darmstadt, 1976.

[16] Murray Shanahan. Solving the Frame Problem: A Mathematical Investigation of the Common Sense Law of Inertia. MIT Press, 1997.

[17] Steve Smale and Ding-Xuan Zhou. Estimating the approximation error in learning theory. Analysis and Applications, 1(1):1-25, 2003.

[18] Douglas Stalker. Grue! the new riddle of induction. Canadian Philosophical Reviews, 15(3):211213, June 1995.

[19] Douglas F. Stalker, editor. Grue! The New Riddle of Induction. Open Court, Peru, 1994.

[20] M.E. Szabo. Polycategories. Communications in Algebra, 3(8):663-689, 1975.

[21] Hudson Turner. A theory of universal causation. Artificial Intelligence, 113:87-123, 1999.

[22] G. Graham White. Intensionality and circumscription. In Salem Benferhat and Enrico Giunchiglia, editors, Proceedings of the 9th International Conference on Non-Monotonic Reasoning (NMR2002), pages 372-379, 2002. Toulouse, April 2002.

[23] G. Graham White. Interpolation for McCain-Turner causal theories. In Proceedings of NRAC'03, 2003.

[24] G. Graham White. Causality, modality and explanation. Notre Dame Journal of Formal Logic, 49(3):313-343, 2008. 